\title{
Оптимизация алгоритма демодуляции сигналов с нелинейным фильтром Шрёдингера при наличии случайной поляризационной модовой дисперсии
}

\author{
И.В. Григоров ${ }^{*}$, Д.В. Мишин \\ Поволжский государственный университет телекоммуникаџий и информатики, г. Самара \\ *E-mail: igor_grigorov@mail.ru
}

DOI:10.31868/RFL2018.137-138

В настоящее время одной из главных проблем передачи сообщений по волоконно-оптическим линиям передачи (ВОЛП) является прием сигналов в условиях действия хроматической дисперсии (ХД), поляризационной модовой дисперсии (ПМД) и нелинейных искажений. В [1] для совместной компенсации ХД и нелинейных искажений был предложен алгоритм последетекторной обработки сигналов, использующий так называемый восстанавливающий нелинейный фильтр Шрёдингера (ВНФШ). Последний представляет собой последовательность нелинейных и линейных фазовых звеньев [2] и является электрическим аналогом ВОЛП, но с сопряженными характерисиками. Алгоритм демодуляции состоит в том, что смесь сигнала и шума на выходе ВОЛП сначала преобразуется ВНФШ, а затем поступает на классический корреляционный демодулятор [3]. Оператор ВНФШ можно обозначить $\mathbf{F}^{-1}$. Он является сопряженным оператору преобразования сигнала в ВОЛП без учета помех.

Рассмотрим модификацию описанного алгоритма приема с учетом ПМД и других случайных параметров ВОЛП. Известно [3], что при синтезе алгоритма приема сигналов в канале со случайно изменяющимися параметрами, оптимальным является байесовский подход, при котором вместо обычного отношения правдоподобия [3] рассматривается усредненное отношение правдоподобия с учетом распределений этих параметров. Строго говоря, все параметры ВОЛП являются случайными, но наиболее существенным здесь является случайный характер параметра $\beta_{1 m}$ для двух ортогонально поляризованных мод $m$-го спектрального канала [4]. Физически этот параметр является обратным групповой скорости соответствующей моды. Вследствие этого, смесь сигнала и шума на выходе ВОЛП, т.е. на входе ВНФШ, будет зависеть не только от времени, но и от указанного параметра.

Применяя байесовский подход для этого случая, можно записать выражение для усредненного отношения правдоподобия с учетом распределения $\beta_{1 m}$ :

$$
\overline{\Lambda\left(\mathbf{Z} / b_{i}\right)}=\int_{\mathrm{B}} w\left(\beta_{1 m}\right) \exp \left\{\frac{2}{N_{0}}\left[\left(\mathbf{F}^{-1}\left(\mathbf{Z}\left(\beta_{1 m}\right)\right), \mathbf{S}_{i}\right)-\frac{E_{i}}{2}\right]\right\} d \beta_{1 m},
$$

здесь $w\left(\beta_{1 m}\right)$ - плотность вероятности указанного параметра, В - его область определения, $\mathbf{F}^{-1}$ - оператор ВНФШ, $\mathbf{Z}\left(\beta_{1 m}\right)$ - вектор смеси сигнала и шума на выходе ВОЛП, зависящий, в том числе, от $\beta_{1 m}, \mathbf{S}_{i}-$ опорные сигналы. Алгоритм демодуляции будет иметь вид: 


$$
b_{j}^{\prime}=\arg \max _{i} \overline{\Lambda\left(\mathbf{Z} / b_{i}\right)}=\arg \max _{i} \int_{\mathrm{B}} w\left(\beta_{1 m}\right) \exp \left\{\frac{2}{N_{0}}\left[\left(\mathbf{F}^{-1}\left(\mathbf{Z}\left(\beta_{1 m}\right)\right), \mathbf{S}_{i}\right)-\frac{E_{i}}{2}\right]\right\} d \beta_{1 m}
$$

здесь $E_{i}$ - энергии сигналов $\mathbf{S}_{i}$. Распределение $w\left(\beta_{1 m}\right)$ практически всегда можно считать гауссовским с известными математическим ожиданием и дисперсией. Описанным способом можно модифицировать алгоритм приема с учетом любого параметра, изменяющегося во времени случайным образом.

Аналитически вычислить интеграл, входящий в (2), сложно, в первую очередь, из-за сложности выражения обратного оператора $\mathbf{F}^{-1}$. Поэтому при цифровой реализации алгоритма (2) указанные интегралы необходимо вычислять приближенно, заменяя непрерывное распределение переменной $\beta_{1 m}$ дискретным.

\section{Литература}

[1] Бурдин В.А., Григоров И.В. Т-Сотт. Телекоммуникачии и транспорт. № 5. С. 18-24 (2013)

[2] Григоров И.В., Широков С.М. Применение теории нелинейных волновых процессов в радиотехнике и телекоммуникациях. - М.: Радио и связь, 2006. -351 с.

[3] Прокис Дж. Цифровая связь. Пер. с англ. // Под ред. Д.Д. Кловского. - М.: Радио связь, 2000. $-800 \mathrm{c}$.

[4] Агравал Г. Нелинейная волоконная оптика. - М.: Мир, 1996. 